\title{
Actividad física, cognición y rendimiento escolar: una breve revisión desde las neurociencias Physical Activity, cognition, and academic performance: a brief review from the neurosciences \\ *Ricardo Ortiz Pulido, **María Luisa Ramírez Ortega \\ *Universidad Veracruzana (México), **Secretaria de Educación de Veracruz (México)
}

Resumen. En este trabajo describimos los efectos que tiene la actividad física sobre el rendimiento académico en niños de edad escolar desde una perspectiva de las neurociencias. También describimos como la actividad física ayuda a la regulación de la neurogénesis y angiogénesis a través de factores neurotrópicos en regiones y estructuras específicas del cerebro, lo cual permite lograr una mejor salud y rendimiento cognitivo en los niños. Considerando el efecto positivo de la actividad física, creemos que los tomadores de decisiones deben generar políticas publicas sustentables para que los niños en edad escolar tengan acceso a la actividad física escolar y extraescolar. Palabras claves: Actividad física, cognición, rendimiento académico, niños.

Abstract. In this paper we describe the effects that physical activity has on academic performance in school-aged children from a neuroscience perspective. We also describe as physical activity improves the regulation of neurogenesis and angiogenesis through neurotropic factors affecting specific regions and structures of brain, which in turn improves health and cognitive performance in children. Considering these positive outcomes, we strongly recommend that stake holders generate sustainable public policies providing reliable access to physical activity during and after the school day for children in school age.

Key words: Physical activity, cognition, academic performance, children.

\section{Introducción}

La actividad física (AF) es cualquier movimiento corporal voluntario que exige un gasto cardiaco mayor al de reposo (Hallal, Azevedo, Reichert, Siqueira, Araújo, \& Victora, 2005). Los efectos benéficos de la AF han sido descritos sobre los sistemas musculo esquelético, cardiovascular y muscular (Blair, Kohl, Gordon, \& Paffenbarger Jr, 1992; Janssen, \& LeBlanc 2010). Tal vez por ello se ha encontrado que llevar a cabo $\mathrm{AF}$ reduce potencialmente la posibilidad de presentar enfermedades crónico-degenerativas que se manifiestan en la edad adulta (Strong, 2005).

La AF se contrapone a la inactividad física. Está última constituye un serio problema de salud pública a nivel mundial (Lee, Shiroma, Lobelo, Puska, Blair, Katzmarzyk, \& Lancet Physical Activity Series Working Group 2012) pues está relacionada con riesgos para la salud, como el aumento de peso corporal (Ng, \& Popkin, 2012).

La inactividad física en la niñez y adolescencia tiene causas y consecuencias. Entre las causas se encuentran que, por ejemplo, los niños pasan muchas horas sentados viendo televisión, usando la computadora (Dietz, 1996; Tammelin, Ekelund, Remes, \& Näyhä, 2007) o jugando video juegos (Marshall, Biddle, Gorely, Cameron, \& Murdey, 2004). Entre las consecuencias destacan obesidad (Strong, 2005), presión arterial alta, diabetes tipo II, enfermedades coronarias del corazón (Daniels, \& Greer, 2008; Hillman, Erickson, \& Kramer, 2008; Hillman, Buck, Themanson, Pontifex, \& Castelli, 2009) y una disminución en la salud cognitiva, así como una reducción en el control ejecutivo y cognitivo (Hillman et al., 2008) y en la función ejecutiva (Diamond, \& Lee, 2011).

En este trabajo revisamos y describimos los beneficios que la AF tiene en los procesos cognitivos y de rendimiento académico en los niños y jóvenes (Bekhechi, \& Khiat, 2019; Flores, Maureira, Diaz, Navarro, Gavotto, \& Matheu 2019;

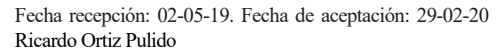

Fraile-García, Terejo-Gonzalez, Esteban-Cornejo, \& Veiga, 2019; Gelabert, Muntaner-Mas, \& Palou, 2019; Rosa, García, \& Carrillo, 2019a; Rosa, García, \& Carrillo, 2019b; Ruiz-Ariza, De la Torre-Cruz, Suárez-Manzano, \& Martínez-López, 2019). Para ello usamos nueva evidencia científica proveniente del uso de dos técnicas: la resonancia magnética (MRI por sus siglas en inglés) y el electroencefalograma (EEG; Hillman; et al., 2014).

\section{Antecedentes}

La AF que promueve el ejercicio aeróbico, ya sea libre, con actividades extraescolares o deporte extraescolar (Strong, 2005), les dan beneficios a los niños y adolescentes que la práctican (Trudeau, \& Shephard, 2008). Por ejemplo, se ha detectado el efecto positivo de las actividades extraescolares en el control cognitivo y en la función ejecutiva (Budde, Voelcker-Rehage, Pietraßyk-Kendziorra, Ribeiro, \& Tidow, 2008; Pesce, Crova, Cereatti, Casella, \& Bellucci, 2009; Best, 2010; Sibley \& Etnier, 2003; Esteban-Cornejo et al., 2015). El control cognitivo se ha definido como el conjunto de procesos asociados a las acciones y su manejo durante el pensamiento (Botvinick, Braver, Barch, Carter, \& Cohen, 2001). Por su parte, la función ejecutiva es considerada como un tipo de rendimiento cognitivo de orden superior, ya que utiliza la creatividad, la flexibilidad, el autocontrol y la disciplina (Diamond, \& Lee, 2011), y sirven para regular los objetivos directos y las interacciones del sujeto con el medio ambiente (Meyer, \& Kieras, 1997).

La relación entre AF-Cognición se ha detectado porque en cada componente, es decir la AF y la cognición, se pueden medir de manera independiente y confiablemente. Por ejemplo, la AF se mide al aplicar de manera controlada la intensidad de la actividad física en niños de jardín (Sirard, 2005; Oliver, Schofield \& Kolt, 2007), primaria (Hollis et al., 2016) y secundaria (Hollis et al., 2017). Por su parte, el rendimiento cognitivo también se puede medir a través de tests fiables (Keeley, \& Fox, 2009). Estos tests evalúan componentes de cognición, tales como tiempo de reacción, atención, memoria y respuesta a estímulos (Rohde, \& Thompson, 
2007), además de control de interferencia y de aptitud aeróbia (Hillman et al., 2009; Smith et al., 2010; Davis et al., 2011). El control de interferencia es uno de los aspectos del control ejecutivo que sirve para evaluar la atención selectiva del control inhibitorio (Hillman et al., 2009). Por su parte, la aptitud aeróbica es la capacidad aeróbica que se determina por el consumo máximo de Oxígeno directo o indirecto (Leger, Mercier, Gadoury, \& Lambert, 1988). Diversos trabajos (Hillman et al., 2009; Smith et al., 2010; Davis et al., 2011) han mostrado que la aptitud aeróbica podría ser fundamental para mejorar tanto el control ejecutivo como el control de la interferencia en la niñez y adolescencia.

La relación AF-Cognición puede explicar varios fenómenos. Por ejemplo, se ha visto que la aplicación de la clase de educación física en niños logra que ellos alcancen un estado de salud y un desarrollo personal social adecuado, además de que produce cambios significativos en su rendimiento académico (e.g. Trudeau, \& Shephard, 2008). Colateralmente, parece que este efecto positivo se mantiene a largo plazo. Esto es porque se ha sugerido que las personas que realizaron AF durante su niñez y adolescencia la siguen haciendo en la edad adulta, permitiendo así sostener y desarrollar su salud física (Ekblom-Bak, Ekblom, Andersson, Wallin, \& Ekblom, 2018) y mental (Hillman, et al., 2008; Hillman et al., 2014), con lo que aumenta la posibilidad de que sean adultos saludables y longevos (Paffenbarger Jr, Hyde, Wing, \& Hsieh, 1986).

A nivel fisiológico se ha detectado que la relación AFCognición es factible. Por ejemplo, hay estructuras específicas del cerebro que son activadas y conectadas en la niñez durante los procesos de control cognitivo (Khan, \& Hillman, 2014) y se creé que la AF modula, mantiene y mejora la salud en el cerebro incrementando la plasticidad neuronal mediante diversas clases de factores de crecimiento (Cotman, \& Berchtold, 2002).

\section{Estado actual del tema}

La AF media los factores neurotrópicos derivados del cerebro (BDNF: siglas en inglés), factor de crecimiento similar a la insulina (IGF-1: siglas en inglés) y factor de crecimiento derivado del endotelio vascular (VEGF: siglas en Inglés) (Neeper, Góauctemez-Pinilla, Choi, \& Cotman, 1995). De acuerdo a la evidencia científica se ha demostrado que la $\mathrm{AF}$ regula el aprendizaje, neurogénesis, angiogénesis a través de una cascada de factores de crecimiento tanto, los BDNF, IGF-1 y VEGF que actúan coordinadamente para mediar los efectos de la AF en el cerebro a partir de fuentes perineales y centrales (Cotman, Berchtold, \& Christie, 2007).

Una preliminar evidencia muestra que la $\mathrm{AF}$ tal vez medie los BDNF (Szuhany, Bugatti, \& Otto, 2015). Los BDNF son una proteína que se ha encontrado en diversas áreas del cerebro como el hipocampo, córtex cerebral, hipotálamo y cerebelo, de igual forma, se ha descrito que la expresión de los BDNF en humanos mediante la técnica de imnunohistoquimica, que localizan y marcan las neuronas en varias estructuras especificas del cerebro (hipocampo, claustrum, amígdala, núcleos de la cama de la stria terminal, septum y los núcleos del tracto solitario (Murer, Yan, \& Raisman-Vozari, 2001). Así mismo, se ha reportado los BDNF en plasma y serum en humanos (Krabbe et al., 2007). Un estudio con niños que se realizó mediante múltiples ensayos en suero, utilizó un análisis en factores de crecimiento (NFG: siglas en inglés) en ese trabajo de investigación se destaca la existencia de una relación longitudinal con respecto a la actividad física vigorosa (Arvidsson, Johannesson, Andersen, Karlsson, Wollmer, Thorsson, \& Dencker, 2018). Además, se ha evidenciado que la AF causa un robusto incremento de neurogénesis en el gyrus dentate del hipocampo de humanos (Van Praag, 2008).

Los BDNF permiten una mejor transmisión, modulación (Ferris, Williams, \& Shen, 2007), plasticidad a nivel neuronal (Ferris et al., 2007; Zoladz, \& Pilc, 2010), regula los niveles de hormonas y neurotransmisores, los cuales reducen el estrés (Shephard, 1997; Taras, 2005; Hillman et al., 2008), autoestima (Shephard, 1997), e incrementa el nivel de endorfinas (Winter, 2007). Así mismo, han sido implicados en desarrollo y funcionamiento neuronal, neurogénesis, crecimiento dendrítico, potenciación a largo plazo de neuronas (Lu, Pang, \& Woo, 2005), plasticidad cerebral (Chaddock et al., 2011a), además se ha explicado la participación de los IGF en la proliferación y diferenciación neuronal y solo a los VEGF en proliferación, ambos factores de crecimiento modulan la proliferación celular endotelial e incrementa el tamaño de los vasos en la región del hipocampo (Cotman et al., 2007).

Se sabe que al realizar AF el músculo promueve que se secrete un IGF-1 que es liberado desde el tejido muscular (Schinder, \& Poo, 2000), lo que permite la inducción de los BDNF y IGF-1 (Cotman, et al., 2007), los BDNF puedan cruzar la barrera hemato-encefalica (Pan, Banks, Fasold, Bluth, \& Kastin, 1998), permitiendo a los BDNF realizar diversas y complejas acciones (Zoladz, \& Pilc, 2010), que se extienden en otras funciones de adquisición y retención (Cotman et al., 2007), y posiblemente puedan ser notados en la maduración del efecto neurotrópico (Lu et al., 2005).

ElBDNF promueve la sinaptogenesis y la diferenciación de nuevas neuronas (Park, \& Poo, 2013). Además se ha demostrado que existe un acoplamiento entre la neurogénesis y la angiogénesis (Palmer, Willhoite, \& Gage, 2000). La neurogénesis es considerado el nacimiento de nuevas neuronas (Gabe et al., 1995), mientras la angiogénesis es la formación de nuevos vasos sanguíneos que permite el aumento del volumen de sangre cerebral (CBV; siglas en inglés) (McDonald, \& Choyke, 2003). Interesantemente, la CBV puede medirse in vivo mediante la técnica de MRI, la cual mapea el volumen regional de sangre en el cerebro (Pereira et al., 2007). Es por ello, que la AF podría ser una estrategia potencial para que pueda inducir mejorías en el estado de ánimo (Stathopoulou, Powers, Berry, Smits, \& Otto, 2006) y cognición (Schmidt, \& Duman, 2010). Debido a los BDNF (Szuhany et al., 2015) que ayudan a mejorar el desarrollo de estructuras y funciones cerebrales (Lu et al., 2005).

En términos de estructura y función cerebral se ha descrito que en la edad escolar quienes realizan actividad física moderada o vigorosa presentan reclutamiento en las regiones del cortex prefrontal y cortex pariental (Chaddock et al., 2012), un mayor volumen en las estructura de hipocampo (Chaddock et al., 2010a), y ganglios basales (Chaddock et al., 2010b), estas estructuras cerebrales tienen funciones específicas (Cohen, Ryan, Hunt, Romine, Wszalek, \& Nash, 1999; 
Culham, \& Kanwisher, 2001; Casey, Jones, \& Hare, 2008; Van Praag, 2008; Hillman et al., 2009), que permiten el control y asociación de diferentes conductas.

El córtex prefrontal es una estructura que está comprometida para el uso del control ejecutivo, respuesta inhibitoria y atención (Hillman et al., 2009), el córtex pariental recae en una categoría denominada córtex de asociación debido a que genera diferentes respuestas con respecto a la localización espacial, control de acción, memoria de trabajo, movimiento de ojos, respuesta de inhibición, cambio de tarea, y calculo (Culham, \& Kanwisher, 2001). La estructura del hipocampo es un área que está implicada en el aprendizaje, la memoria (Van Praag, 2008) y memoria relacional (que significa, habilidad de integrar elementos, lugares, objetos a lo largo del tiempo y en el espacio temporal) (Cohen et al., 1999). Además, esta estructura en humanos participa en la formación de nueva memoria relacional y procesos que requieren relacionar un simple objeto, dependen del circuito parahipocampal (Brassen, Weber-Fahr, Sommer, Lehmbeck, \& Braus, 2006), la posible asociación de un alto nivel de aptitud física y el nivel de la habilidad superior de trabajar procesos relacionados en rendimiento de memoria y el volumen de hipocampal sugiere una fuerte habilidad cognitiva de control ejecutivo y uso de memoria que posiblemente participa la vía de interacción prefrontal hipocampal (Chaddock, Hillman, Buck, \& Cohen, 2011b). Otra de las estructuras que esta críticamente involucrada son los ganglios basales, que es una estructura asociada a cambios de respuestas en preparación, atención e inhibición, esta estructura cerebral se subdivide en dorsal-striatum y ventral-striatum, la primer subregión está implicada en estímulos y retos, atiende a seleccionar respuestas motoras, de cognición y ejecución de conductas de aprendizaje, la segunda subregión está relacionada con la vía de recompensa, reforzamiento del aprendizaje y estado de motivación (Casey et al., 2008).

Todas estas estructuras del cerebro que hemos mencionamos anteriormente son componentes necesarios del control cognitivo y rendimiento académico. Además, existe una asociación entre la AF, la aptitud aérobica y las estructuras neuronales, lo cual podría permitir la percepción adicional de la existencia de una relación entre la AF y la cognición en niños (Chaddock et al., 2011a) y rendimiento académico (Buck, Hillman, \& Castelli, 2008; Cancela-Carral, Pérez, \& Espiño, 2016).

Diversas revisiones sistemáticas observacionales han sido realizadas con el fin de demostrar como la AF ha sido asociada a mejorar el rendimiento académico, (Strong et al., 2005; Rasberry, Lee, Robin, Laris, Russell, Coyle, \& Nihiser, 2011; Singh, Uijtdewilligen, Twisk, Van Mechelen, \& Chinapaw, 2012; Mura, Vellante, Nardi, Machado, \& Carta, 2015). El rendimiento académico se puede diagnosticar mediante evaluaciones estandarizadas en la escuela, como son las matemáticas o la habilidad de leer (Roberts, Freed, \& McCarthy, 2010), estas han sido realizadas considerando el grado y edad (Chomitz, Slining, McGowan, Mitchell, Dawson, \& Hacker, 2009), también esta habilidad depende del conocimiento del niño con respecto a los que adquiere en casa o del medio ambiente tanto como la calidad y cantidad de instrucción académica que la niñez recibe en la escuela (Keeley, \& Fox, 2009).
De esta forma analizar el impacto de la AF y rendimiento académico merece la atención de las neurociencias para analizar y describir los avances existentes. Un trabajo de investigación que evaluó la comprensión lectora utilizó tomografía por emisión de positrones (PET; sigla en Inglés) en este estudio se demostró que la actividad cerebral fue mayor en la región de cortex prefontal y cortex cingulado (Maguire, Frith, \& Morris, 1999). Con respecto a los procesos de cálculos matemáticos y el cerebro estos han sido vinculado a regiones bilaterales (intrapariental sulcus) en niños y adultos (Ansari, \& Dhital, 2006). Sin embargo, se ha examinado el neuro-desarrollo evaluando cálculos aritméticos en niños y jóvenes los resultados de este estudio demostró que existió, reclutamiento y especialización del cortex pariental inferior derecho (Rivera, Reiss, Eckert, \& Menon, 2005). Dado que la comprensión lectora como las matemáticas obtienen activación en la red fronto-pariental (Maguire et al., 1999; Rivera et al., 2005), esto permite la posibilidad de examinar la relación entre aptitud física y el rendimiento académico que también ha sido relacionada con la red fronto-pariental (ChaddockHeyman et al., 2015), así al utilizar técnicas especializadas en el análisis del cerebro y del cuerpo se han reportado beneficios fisiológicos de la AF para la salud del cuerpo y cerebro (Strong et al., 2005; Hillman et al., 2008; Hillman et al., 2014).

Por lo que, nuestro trabajo está orientado a unir el conocimiento de la AF, cognición y rendimiento académico desde la perspectiva de la neurociencia, Además la evidencia científica en este trabajo sobre la actividad física de alta intensidad y los estudios de neuro-imagen en niños, niñas y adolescentes sugieren la posible modulación de circuitos del cerebro que se relacionan con el control cognitivo, lo que ha permitido proponer que altos niveles de aptitud aeróbica puede estar relacionados con un adecuado rendimiento académico durante la niñez, con esto, se podría crear no solo un impacto positivo en la salud de órganos, sistemas, si no también efectos estructurales en el cerebro de los niños, niñas y jóvenes.

En este sentido nosotros describimos 10 trabajos científicos de acuerdo a la metodología empleada en este trabajo con el objetivo de describir la existencia de la actividad neuronal en estructuras cerebrales vinculadas a la AF cognición y rendimiento académico en niños y jóvenes.

\section{Metodología}

Nuestros criterios de inclusión fue realizar una selección, identificación, detección, elegibilidad de estudios de forma sistemática que se describe en la figura 1 con el objetivo de tener una mejor comprensión de describir este trabajo, a través de la base de datos electrónica disponible, se realizó una búsqueda exhaustiva se incluyó literatura académica investigada en SPORTDiscus, Psycinfo, Scopus y MEDLINE, partir del primer registro hasta del 26 de julio del 2018. La estrategia para la investigación consistió en cinco elementos académicos que fueron descritos en niños y jóvenes desde la perspectiva de la neurociencia: (1) actividad física, (2) cognición, (3) rendimiento académico (4) población, (5) neuro-imagen y electroencefalograma. Se investigaron subcategorías para cada uno de estos elementos. (1) «condición física»; «ejercicio físico»; «aptitud aeróbica»; aptitud física»; «activi- 
dad física moderada y vigorosa»; «actividad física al aire libre»; «ejercicio extraescolar»; «deporte extraescolar»; (2) «nivel cognitivo»; "control ejecutivo»; «función ejecutiva»; «rendimiento cognitivo»; función cognitiva; «tiempo de reacción»; «atención»; «memoria»; «control de interferencia»; «control inhibitorio»; «control cognitivo»; (3) «logro académico»; «aprendizaje»; «evaluaciones estandarizadas»; habilidad para leer»; «conocimiento matemático»; (4) «niños»; «niñas»; «adolescentes»; (5) «tomografía por emisión de positrones»; imágenes de resonancia magnética»; «electroencefalograma»; «difusión de tención de imágenes»; volumen de sangre cerebral»; «neurociencias».

Todos los estudios elegidos e incluidos fueron revisados en el idioma inglés que se ajustaron a la descripción disponible antes mencionada. En esta revisión de la literatura, la lista de referencias de los artículos identificados fueron consultados e identificados para efectos de la investigación, estos fueron incluidos si existía una relación de la actividad cerebral entre AF, cognición o rendimiento académico en niños, niñas o jóvenes saludables. Los criterios de exclusión, fueron eliminar resúmenes y tesis que hayan considerado a una población que tuviera problemas de desórdenes de aprendizaje o lenguaje.

Los datos relacionados con los participantes (media de edad, sexo, aptitud aeróbica o condición física que fue representada por el volumen máximo de Oxígeno (VO2max)), u otros valores representativos como distancia ó tiempo, sobre los estudios (diseño, tamaño de muestra, tipo de intervención).

Nosotros identificamos trabajos en la base de datos y adicionalmente los incluimos, posteriormente eliminamos las duplicaciones. Además, excluimos todos los artículos que no tuvieron los criterios de inclusión. Finalmente los artículos incluidos para este estudio fueron 10 trabajos de investigación que demuestran como la AF causa cambios morfológicos y funcionales en estructuras específicas del cerebro y produce un robusto efecto en la cognición o rendimiento académico.

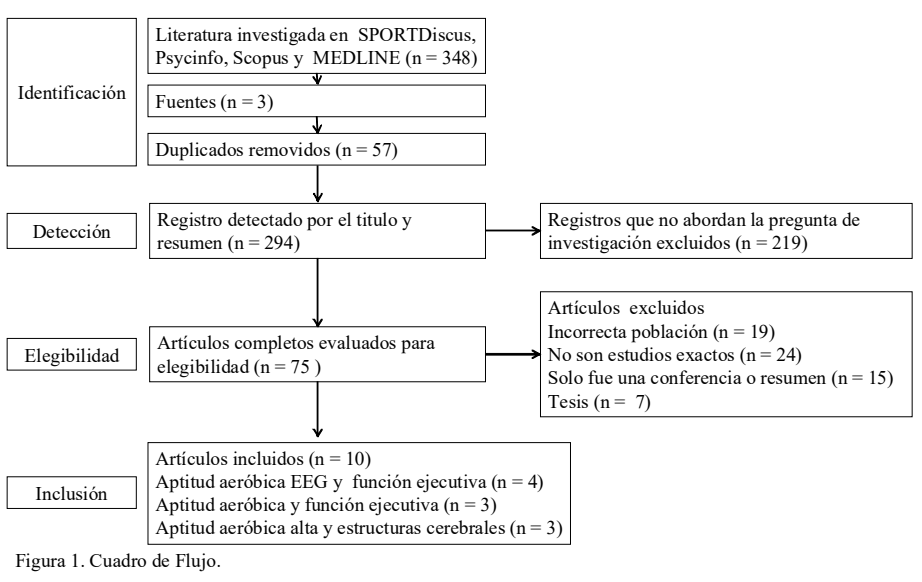

Resultados

Después de analizar y eliminar duplicados o ilegibles artículos, nosotros identificamos 10 artículos publicados entre 2005 al 2014 elegibles, los artículos relevantes muestran sus resultados descritos detalladamente en la Tabla 1, 2 y 3.

De los 10 artículos identificados en esta revisión sobre $\mathrm{AF}$, cognición y rendimiento académico, solo uno ha sido identificado de intervención que incluyó examinar a los niños con EEG. Durante este estudio un grupo fue asignado a un programa de AF de 2 horas (al menos de 70 minutos de MVPA) después de clases (Hillman et al., 2014), de igual forma, analizamos tres documentos más, en ellos no existió intervención en los niños y niñas con EEG ó MRI (Hillman et al., 2005; Hillman et al., 2009; Pontifex et al., 2011) uno de los tres documentos analizó a sujetos adultos y niños (Hillman et al., 2005), así mismo, tres trabajos de investigación fueron examinados mediante MRI (Chaddock et al., 2010a; Chaddock et al., 2010b; Chaddock et al., 2012) y solo tres documentos descritos en este trabajo no utilizaron EEG ó MRI (Buck et al., 2008; Wu, Pontifex, Raine, Chaddock, Voss, Kramer, \& Hillman, 2011; Hillman et al., 2014).

En estos 10 estudios incluidos participaron un total de 635 (324 niños y 311 niñas). En cinco estudios incluidos se examinó la aptitud aeróbica alta 53.1 y baja $35.2 \mathrm{ml} / \mathrm{kg} / \mathrm{min}$, mediante análisis de pruebas de laboratorio (Chaddock et al., 2010a; Chaddock et al., 2010b; Chaddock et al., 2011; Pontifex et al., 2011; Chaddock et al., 2012). En dos trabajos se analizó la aptitud aeróbica al medir los lapsos de los recorridos (20 metros de ida y vuelta) (siglas en Inglés PACER laps), (la aptitud aeróbica alta $31.2 \mathrm{ml} / \mathrm{km} / \mathrm{min}$ y la baja $11.1 \mathrm{ml} / \mathrm{km} / \mathrm{min}$ ) (Hillman et al., 2005; Hillman et al., 2009). Igualmente, describimos dos estudios, sin categorizar la aptitud aeróbica alta 0 baja, el primero solo muestra el promedio de la aptitud aeróbica $21.73 \mathrm{ml} / \mathrm{kg} / \mathrm{min}$ (Hillman et al., 2014) y el segundo trabajo fue realizado con 74 niños categorizado, por edades y la media estadística de PACER laps (Buck et al., 2008).

En los 10 estudios que se investigaron las variables demográficas. Se evaluó el estatus demográfico y socioeconómico (SES: siglas en inglés) y se examinó Kaufman Brief Intelligence Test (K-BIT:siglas en Inglés) (Hillman et al., 2005; Buck et al., 2008; Hillman et al., 2009; Chaddock et al., 2010a; Chaddock et al., 2010b; Chaddock et al., 2011b; Pontifex et al., 2011; Wu et al., 2011; Chaddock et al., 2012; Hillman et al., 2014). El SES se caracteriza por el índice de tres variables: 1) la participación en forma gratuita o programa de almuerzo a precio reducido en la escuela, 2) el nivel más alto de educación de los padres y 3) el número de padres que trabajan tiempo completo (Birnbaum, Lytle, Murray, Story, Perry, \& Boutelle, 2002). En el K-BIT se obtuvieron mediciones del score compuesto del coeficiente intelectual (IQ; siglas en Inglés), K- BIT score cristalizado (vocabulario) y KBIT score fluido (matrices) (Kaufman,1990). En los 10 trabajos de investigación no mostraron diferencias significativas entre los grupos que se contrastaron.

Solo en seis trabajos de investigación se midió la escala de Tanner que evaluó el estatus puberal (Tanner: Siglas en inglés) (Taylor, Whincup, Hindmarsh, Lampe, Odoki, \& Cook, 2001; Chaddock et al., 2010b; Chaddock et al., 2011b; Pontifex, et al., 2011; Wu et al., 2011 Hillman et al., 2014). Con respecto a la escala de atención de desórdenes por déficit de hiperactividad (ADHD: Siglas en Inglés) (DuPaul, Power, Anastopoulos, \& Reid, 1998; Birnbaum et al., 2002), siete estudios utilizaron la esca- 
la V (Hillman et al., 2009; Chaddock et al., 2010a; Chaddock et al., 2010b; Chaddock et al., 2011; Pontifex et al., 2011; Wu et al., 2011; Chaddock et al., 2012) y un solo estudio ocupo la escala IV para evaluar a los niños respectivamente (Hillman etal., 2014).

La aptitud física aeróbica fue examinada en todos los trabajos incluidos. En tres de ellos fue mediante FITNESSGRAM los test incluyeron, la aptitud muscular (fuerza muscular de extremidades superiores, fuerza abdominal, aptitud de flexibilidad (test de flexibilidad de sentado), composición corporal (IBM;siglas en inglés) (Plowman, \& Meredith, 2013) que incluyo la prueba de PACER laps (es una prueba de campo que se llevó acabo recorriendo una distancia de $20 \mathrm{~m}$. de ida y vuelta, el sujeto corrió tan lejos como sea posible, con incrementos de velocidad minuto (Leger et al., 1988; Hillman et al., 2005; Buck et al., 2008; Hillman et al., 2009) y en seis investigaciones fue analizado la aptitud física aeróbica (Chaddock et al., 2010b; Chaddock et al., 2011; Pontifex et al., 2011; Wu et al., 2011; Chaddock et al., 2012; Hillman et al., 2014) utilizando una banda rodante en el test modificado de Balke, es un test de laboratorio que midió el percentil que expresa, el score de valores normativos basados en la edad y sexo para el V02max (Siglas en Inglés: VO2maxpercentil:) (Shvartz, \& Reibold, 1990), antes y duran-

Tabla 1

\begin{tabular}{|c|c|}
\hline $\begin{array}{c}\text { Autor/País } \\
\text { Características sujetos }\end{array}$ & Resultados \\
\hline (Hillman et al., 2005) & \\
\hline Estados Unidos & $\begin{array}{l}\text { El analisis de los eventos relacionados con los potenciales del cerebro en formas de onda indican que en adultos exhibe una larga amplitud de P3, Cz, Pz, y una disminucion de } \\
\text { la amplitud Oz cuando fue comparado con los niños }\end{array}$ \\
\hline 24 niños (9.6 años de edad) & $\begin{array}{l}\text { la amplitud } \mathrm{OZ} \text { cuando fue comparado } \\
\qquad(t(1,49=2.8, p<.008) .\end{array}$ \\
\hline 27 adultos (19.3 años de edad) & Los análisis indicaron que la aptitud física alta en niños exhibió una alta amplitud en P3 comparada con los tres grupos \\
\hline $\begin{array}{l}\text { Aptitud fisica alta } 12 \text { (7 niños) } \\
\text { Antitud fisica baia } 12(6 \text { ninos) }\end{array}$ & $(t(1,22=3.7, p<.001)$ \\
\hline 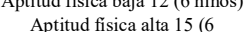 & Los adultos tuvieron una latencia rápida en la amplitud $\mathrm{Cz}$ y, Pz comparado con los niños \\
\hline $\begin{array}{l}\text { Aptitud fisica alta } 15(6 \\
\text { hombres) }\end{array}$ & $(t(1,49=3.0, p<.004)$ \\
\hline Aptitud física baja 12 (7 & Los adultos tuvieron mejor reacción rápida (media $=391.1 \mathrm{~ms} \mathrm{SE}=9.5$ ) que los niños. \\
\hline hombres) & Y la comparación de los niños con aptitud aeróbica alta tuvieron mejor reacción (media = 465.2 ms SE = 9.5 que los niños con aptitud aeróbica baja. \\
\hline (Hillman et al., 2009) & \\
\hline Estados Unidos & Existió un efecto de la aptitud fisica de los grupos congruente e incongruente de la tarea del test de flanker que fueron observados \\
\hline $\mathrm{n}=38$ & $F(1,36)=5.0, p .<03, \eta^{2}=.12$ \\
\hline Aptitud aeróbica alta & Responde mejor a los participantes de alta aptitud $(M=82.1 \pm 10.2 \%)$ fisica que los de baja $(M=75.2 \pm 9.9 \%)$. \\
\hline 19 (9 niñas) & El análisis de $\mathrm{P} 3$ con amplia amplitud revelo que el efecto en la condición física $F(1,36)=4.7, p<.04, \eta^{2}=12$ tuvo mayor amplitud en el grupo de mayor aptitud física con ( $M$ \\
\hline años de edad & $=9.4 \pm 2.4 \mu \mathrm{V})$ respecto de grupo de menor aptitud física $(M=7.6 \pm 2.7 \mu \mathrm{V})$. \\
\hline $9.3(0.9)$ & La media de la amplitud P3 demostró la más alta amplitud $F(1,36)=4.9, p<.04, \eta^{2}=12$ \\
\hline Aptitud aeróbica baja & En el grupo de condición física alta $(M=5.1 \pm 3.5 \mu \mathrm{V})$, comparado con el de baja $(M=3.3 \pm 3.5 \mu \mathrm{V})$. \\
\hline 19 (9 niñas) & El análisis separado de cada uno de los grupos indica que los participante con alta condición exhibe una alta correlación del error posterior a la respuesta exacta de la amplitud \\
\hline $\begin{array}{l}\text { Años de edad } \\
9.5(1.0)\end{array}$ & $\mathrm{P}_{\mathrm{C}} r=.51, p<.03$, sin embargo en los participantes con baja condición $r=.33, p=.16$, no fueron observadas. \\
\hline (Pontifex et al., 2011) & \\
\hline Estados Unidos & Los resultados demuestran un efecto en la respuesta de exactitud para el grupo de baja aptitud aeróbica $(75.9 \pm 1.8 \%)$ y relativo a el grupo de alta aptitud aeróbica $(84.8 \pm 1.8 \%)$. \\
\hline $\mathrm{n}=24$ & itud física baja aumenta la r \\
\hline $\begin{array}{l}\text { Aptitud aeróbica alta } \\
10 \text { niñas y } 14 \text { niños }\end{array}$ & $\begin{array}{l}\text { Los resultados neuro-electricos muestran el promedio de la EPR formas de onda, que se midieron con diferentes electrodos se midieron amplitudes y latencias al comparar el } \\
\text { grupo de aptitud aeróbica alta contra el grupo aptitud aeróbica baja con la interacción de congruencia e incongruencia del flanker test } \mathrm{N} 2 \text { mostro un incremento en la amplitud }\end{array}$ \\
\hline Años de edad 10.0 (.6) & solo en los sitios de los electrodos: centro-pariental, pariental y pariento- occipital $[\operatorname{ts}(47)=3.1, p=.003]$. \\
\hline $\mathrm{n}=24$ & $\mathrm{~N} 2$ mostro una larga y baja latencia [ts $(47)=2.0, p=.057]$. \\
\hline Aptitud aeróbica baja & P3 revela efectos de disminución de la amplitud en la tarea compatible $[\mathrm{t}(46)=3, p=.01]$. \\
\hline Años de edad 10.1 (.6) & P3 mostro una larga y baja latencia a nivel central, centro-pariental y pariento-occipital [ts(46)=2.9, $p=.006]$. \\
\hline 13 niñas y 11 niños) & \\
\hline & El pulso (frecuencia cardiaca por minuto durante las sesiones fue de $137( \pm 15.1)$ y realizaron 4246 pasos $( \pm 1039.9)$. \\
\hline (Hillman et al., 2014) & Solo existieron cambios en el grupo de intervención de aptitud aeróbica pre y post (5.5 percentil, $95 \%$ CI: 1.9 to $9.1, \mathrm{~d}=.42$ ). \\
\hline Estados Unidos. & Ambos grupos incrementaron el IMC, sin embrago el grupo control mostro más incremento $(0.5 \mathrm{~kg} / \mathrm{m} 2,95 \%$ CI: 0.2 to $0.9, \mathrm{~d}=.3$ \\
\hline $\mathrm{n}=221$ & revelaron efectos en la inhibición atencional en ambos grupos. El grupo intervención mostro el incremento de la amplitud P3 (1.4 mV, $95 \%$ CI: 0.3 to $2.6, d=.34)$ En la \\
\hline $\begin{aligned} \mathrm{n}= & 109 \text { grupo intervención } \\
& \text { niñas } 53 \text { niños } 56\end{aligned}$ & El grupo intervención demostró una rápida latencia en la condición del incongruente flanker test (20.1 millisegundos, $95 \%$ CI: 2.6 to $37.6, \mathrm{~d}=.31$ ), así como el grupo control \\
\hline $\mathrm{n}=112$ grupo lista de control & $\begin{array}{l}\text { ( } 32.0 \text { millisegundos, } 95 \% \text { CI: } 6.9 \text { to } 57.2, \mathrm{~d}=.34 \text { ). } \\
\text {. }\end{array}$ \\
\hline niñas 53 niños 56 & $\begin{array}{l}\text { Ambos grupos mostraron cambios en la flexibilidad cognitiva pre y post en los grupos homogeneo y heterogeneo, el incremento en el rendimiento en el grupo heterogéneo sobre } \\
\text { las tareas fue mejor }(4.8 \%, 95 \% \text { CI: } 1.1 \text { to } 8.4, \mathrm{~d}=.35) \text {. }\end{array}$ \\
\hline $\begin{array}{l}\text { No existieron diferencias con } \\
\text { respeto a la intervención y la }\end{array}$ & Un incremento en la amplitud P3 de los ensayos heterogéneos a partir del test y pos-test fue observado solo en el grupo intervención p ( $1.5 \mathrm{mV}, 95 \% \mathrm{CI}: 0.6$ to $2.5, \mathrm{~d}=.43$ ) \\
\hline $\begin{array}{l}\text { respeto a la intervencion y la } \\
\text { lista de control de grupos. }\end{array}$ & Existió una correlación positiva en el FITKids con el cambo de amplitud $(\mathrm{r}=0.22, p=.02)$ y negativamente correlacionad con el cambio de latencia en $\mathrm{P} 3(\mathrm{r}=20.22, p=.02)$. \\
\hline & $\begin{array}{l}\text { Una similar relación fue observada en la conducta de cambio de tarea que se correlaciono con el cambio de tarea en condición heterogénea más exigente necesitando una mayor } \\
\text { inhibición, memoria de trabajo y flexibilidad cognitiva }(\mathrm{r}=.24, p=.01) \text {. }\end{array}$ \\
\hline
\end{tabular}

Tabla 2

\begin{tabular}{|c|c|}
\hline $\begin{array}{c}\text { Autor/País } \\
\text { Características de los sujetos }\end{array}$ & Resultados \\
\hline $\begin{array}{c}\text { (Buck et al., 2008) } \\
\text { Estados Unidos } \\
\text { Todos los participantes } 74 \text { ( } 41 \text { niños) } \\
7 \text { a } 12 \text { años de edad }(9.3 \text { media; } 1.4 \text { DE) } \\
\text { Grupo } 7 \text { a } 8 \text {, años de edad } \\
22 \text { (11 niños) } \\
\text { Grupo } 9 \text { a } 10 \text {, años de edad } \\
39 \text { (23 niños) } \\
\text { Grupo } 11 \text { a } 12 \text {, años de edad } \\
13 \text { (6 niños) }\end{array}$ & $\begin{array}{l}\text { Los niños y niñas con más edad y un alto coeficiente intelectual (IQ) están asociados a un mejor rendimiento } \\
\text { Análisis de regresión } \\
\qquad \begin{array}{r}R^{2}=.38, F(3,70)=16.0, p<.001 \\
\text { La aptitud aeróbica está asociado con el mejor rendimiento } \\
\text { Análisis de regresión } \\
p r=.27, t(69)=2.4, p=.02, \beta=.25\end{array} \\
\end{array}$ \\
\hline $\begin{array}{l}\text { (Wu et al., 2011) } \\
\text { Estados unidos } \\
\mathrm{n}=48 \\
\text { Aptitud fisica alta } \\
24(10 \text { niños }) \\
\text { Años de edad } 10.0 \text { (.6) } \\
\text { Aptitud física baja } \\
24 \text { (13 niños) } \\
\text { Años de edad } 10.1 \text { (.6) }\end{array}$ & $\begin{array}{c}\text { Existieron diferencias significativas con respecto de la aptitud aeróbica alta en VO2max } \\
t(1,46)=12.2, p<.001, \text { percentil } \\
t(1,46)=54.8, p<.001\end{array}$ \\
\hline $\begin{array}{l}\text { (Chaddock et al., 2011) } \\
\text { Estados Unidos } \\
\mathrm{n}=22 \\
\text { Aptitud aeróbica alta } \\
10 \text { niñas y } 12 \text { niños } \\
\text { Años de edad } 9.9(.5) \\
\mathrm{n}=24 \\
\text { Aptitud aeróbica baja } \\
13 \text { niñas y } 11 \text { niños } \\
\text { Años de edad } 9.9(.6)\end{array}$ & $\begin{array}{l}\text { Los análisis estadísticos evidenciaron que la aptitud aeróbica baja muestra bajo rendimiento académico en comparación con el grupo de aptitud alta } \\
\qquad \begin{array}{l}\text { especialmente en los códigos que están relacionados } \\
\qquad F(1,43)=5.5, p=0.02, \eta^{2}=.113\end{array}\end{array}$ \\
\hline
\end{tabular}


te la prueba de esfuerzo (VO2max) se registró la frecuencia cardiaca en un monitor polar. Al final de la prueba de esfuerzo se evaluó el índice de esfuerzo percibido (IEP) con la escala para niños y niñas evaluando el caminar y correr (Utter, Roberson, Nieman, Kang, 2002). Solo en uno de ellos no fue claramente descrito, solo muestran los datos de VO2max de los sujetos (Chaddock et al., 2010a).

El control ejecutivo se evaluó mediante la función cognitiva y de memoria, esta fue valorada en seis estudios mediante el flanker test (Hillman et al., 2009; Chaddock et al., 2010b; Pontifex et al., 2011; Wu et al., 2011; Chaddock et al., 2012; Hillman et al., 2014) y en cuatro estudios fueron evaluados mediante cuatro test diferentes, visual oddball, (Hillman et al., 2005) tarea de color, (Buck et al., 2008) tarea de memoria, reconocimiento de memoria (Chaddock et al., 2010a) y un test de memoria de Henke (Chaddock et al., 2011). Un único estudio utilizó dos evaluaciones, la primera fue la versión modificada del flanker test y cambio de tarea de color, la primer prueba examinó aspectos de control inhibitorio neuroelectrico e índices de conductas del rendimiento, y el segundo test evalúo aspectos de memoria cognitiva y flexibilidad cognitiva (Hillman et al., 2014).

En la tabla 1. Se muestran 4 de los trabajos de investigación relacionados con la aptitud aeróbica, EEG y la función ejecutiva. En la tabla 2. Se describen 3 investigaciones que revelan los efectos de la aptitud aeróbica y función ejecutiva. Y en la tabla 3 se observan tres estudios que muestran una asociación entre la aptitud aeróbica alta, estructuras cerebrales (hipocampo, ganglios basales y LMFG, RMFG, SMA, ACC, LSPL) y la función ejecutiva.

\section{Conclusiones}

Nosotros identificamos y revisamos 10 estudios relevantes a la $\mathrm{AF}$, cognición y rendimiento académico de los cuales cuatro estudios aquí descritos evidenciaron la relación de la aptitud aeróbica y EEG. Estos estudios mostraron los efectos significativos en el componente endógeno P3 (Hillman et al., 2005; Hillman et al., 2009; Pontifex et al., 2011; Hillman et al.,
2014) que se relaciona con eventos potenciales del cerebro (EPR: siglas en inglés), y reflejan cambios de voltaje que ocurren en respuesta a la preparación de estímulos (Polich, Ladish, \& Burns, 1990).

Los descubrimientos encontrados (Hillman et al., 2005) indican que los niños con una aptitud física alta tienen una gran amplitud de voltaje P3 comparada con baja aptitud en niños y en los otros grupos, lo cual sugiere que existe una gran población de neuronas que han sido reclutadas para las tareas específicas. Así mismo, el grupo con aptitud física alta obtuvo un proceso de velocidad cognitivo más rápido comparado con el grupo de aptitud física baja (es decir, P3 latencia) independientemente de la condición de la tarea o grupo de tareas. Adicionalmente los niños con aptitud física alta exhiben menor tiempo de reacción que los niños con aptitud física baja. Similares resultados se encontraron (Hilman et al., 2009) en relación a la amplitud de voltaje P3 que sugiere que la aptitud aeróbica está relacionada con la función cognitiva en preadolescentes, además los datos sugieren que la aptitud aeróbica alta es beneficiosa para las tareas de rendimiento, mientras otro estudio (Pontifex et al., 2011) encontró que el grupo de alto nivel de aptitud aeróbica tienen la posibilidad de un mejor control del proceso cognitivo. De igual forma, otra investigación (Hillman et al., 2014) evidencio que el grupo interferencia (que realizó AF con niños y niñas durante 9 meses) mostró la existencia de un incremento en la amplitud P3 a partir del pre-test y postests. Así mismo, existió una correlación positiva en el FITKids con la amplitud P3 y negativamente correlacionado en latencia en P3. Este estudio manifestó incrementos en la aptitud aeróbica, conductas y control ejecutivo, por lo que parece ser que la aplicación de actividad fisca tiene una alta relación con los cambios en los índices de atención, proceso de velocidad y el rendimiento ejecutivo. Por ello se sugiere la participación diaria de programas de actividad física. En resumen, los resultados permitieron discriminar las tareas, así mismo, se encontró que la aptitud aeróbica alta está asociada a actividad neuro-eléctrica que permite analizar en el grupo con aptitud aeróbica alta una larga la amplitud P3 y un 
rápido tiempo de reacción en las funciones neuro-eléctricas. Se cree que el grupo con aptitud aeróbica alta tal vez fue capaz de reclutar recursos neurales para mejorar el rendimiento de las tareas propuestas.

Nosotros examinamos tres estudios que muestran la relación de la AF y el control ejecutivo los cuales no utilizaron EEG ó MRI. Para analizarlos se utilizaron diferentes test, el primero estudio utilizó el stroop test, que examinó interferencia de control, (que es uno de los aspectos del control ejecutivo, utilizado para la evaluación de la atención selectiva del control inhibitorio), los resultados encontrados demuestran que los niños con más edad están asociados a un mejor rendimiento, además que los que tuvieron mejor aptitud aeróbica tienen un mejor rendimiento, es por ello que, estos hallazgos sugieren que la aptitud aeróbica talvez pueda beneficiar la cognición durante la adolescencia y una asociación a un mejor rendimiento académico (Buck et al., 2008). En el segundo estudio (Wu et al., 2011) se utilizó el flaker test, que examina la interferencia (es un aspecto del control ejecutivo), de acuerdo a sus resultados y descubrimientos se propone, que la aptitud física está asociada al mejor rendimiento cognitivo durante la adolescencia. El tercero estudio investigado (Chaddock et al., 2011) utilizó el test de memoria de Henke, los resultados muestran que el grupo de aptitud física baja no obtuvo resultados favorables en el reconocimiento del rendimiento de memoria en comparación con el grupo de aptitud aeróbica alta quienes relacionaron y codificaron mejor las caras de las personas y las casas que recuerdan cuando les fue aplicado el test, esto confirmó las predicciones realizadas sobre el uso de la memoria relacional, es decir, probar que los niños con baja condición tiene más dificultad para la utilización del control ejecutivo y el proceso de la memoria relacional.

Nosotros describimos tres estudios que utilizaron MRI. El primero investigó sobre la asociación de la aptitud física alta y baja y sus efectos de actividad cerebral en el área del hipocampo, además utilizó un test de memoria de reconocimiento y relacional. Los resultados demostraron que el volumen hipocampal fue asociado a la tarea de rendimiento, pero no así para los ítems de memoria, a partir de ahí los autores propusieron un modelo de mediación bilateral del volumen hipocampal que muestra mediación de la relación entre el nivel de aptitud aeróbica y memoria relacional (Chaddock et al., 2010a).

En otro estudio se exploró la aptitud física alta, aptitud física baja y la función de los ganglios basales mediante la utilización MRI, además se evaluó con un test de control ejecutivo (flanker test), los resultados mostraron diferencias significativas entre el grupo aptitud aeróbica alta y el grupo aptitud baja con respecto al volumen del dorsal striatum de los ganglios basales y el flanket test. Se sabe que esta área cerebral está involucrada en el control cognitivo, integración motora y respuesta de resolución. Sin embargo, no existió asociación entre la aptitud aeróbica baja y alta en el test flaker (conducta de interferencia) y el volumen del dorsal striatum que involucra el núcleo acumbes involucrado a niveles límbicos y procesos de recompensa (Chaddock et al., 2010b). Los hallazgos proponen que un gran volumen dorsal stratital está relacionado con el control cognitivo.

El tercer estudio exploró la actividad cerebral con MRI en niños con aptitud física alta y baja y el vínculo que tiene con el control cognitivo durante la función ejecutiva (flanker test), los resultados demostraron que la aptitud aeróbica fue relacionada con la función cognitiva en niños. Los datos del MRI sugieren que la aptitud aeróbica permite la activación de áreas frontal y pariental durante el primer bloque de tareas del flanker test. Cuando las condiciones del test requirieron más concentración solo el grupo de aptitud alta tuvo mayor exactitud en sus respuestas durante las condiciones incongruentes del flanker test, al realizar el análisis con MRI se demostró que existió mayor reclutamiento de las áreas prefrontal y pariental (Chaddock et al., 2012).

Las investigaciones descritas aquí con EEG indican una relación con la aptitud aeróbica y función ejecutiva (Hilman et al., 2014), otros trabajos que han utilizado MRI revelan efectos positivos entre la aptitud aeróbica y el control ejecutivo (Buck et al., 2008), así mismo, diferentes estudios han probado la asociación entre de la aptitud aeróbica y estructuras cerebrales (Chaddock et al., 2010a; Chaddock et al., 2010b; Chaddock et al., 2012). Además estos hallazgos encontrados sobre la aptitud fisica aeróbica alta en niños comparada con los de baja aptitud indica que existe un grupo de neuronas que está siendo reclutadas para esta tarea a través de proceso neuro-cognitivo más rápido, logrando así responder con menos latencia a estímulos de atención y trabajo de memoria. Lo cual provee de nuevos datos para los investigadores, al considerar que esta información científica permitiría desarrollar nuevas líneas de trabajo de investigación.

De igual forma se han efectuado diferentes revisiones sistemáticas observacionales sobre los efectos y asociaciones existentes, se ha demostrado que aumenta los niveles de concentración, atención del aprendizaje cognitivo (Scheuer, \& Mitchell, 2003; Strong et al.,2005; Rasberry et al., 2011; Sing et al., 2012; Mura et al., 2015). Al analizar la AF y el rendimiento escolar por grados y conductas en la clase se reportó que no afecta el tiempo realizado en la instrucción de la educación física de la escuela, la AF o los programas de deporte (Trudeau, \& Shephard, 2008). Además, un incremento de AF en el currículo escolar no influye en la disminución del rendimiento académico (Hillman et al., 2008). Sin embargo, otra revisión, demostró una débil correlación o la no existencia, entre el nivel de actividad física y el nivel de rendimiento académico (Tremblay, Inman, \& Willms, 2000; Coe, Pivarnik, Womack, Reeves, \& Malina, 2006; LeBlanc et al., 2012; Syväoja, Kantomaa, Ahonen, Hakonen, Kankaanpää, \& Tammelin, 2013). Lo cual pone de manifiesto que se debe de hacer más investigación para lograr entender mejor el impacto de la actividad física y el rendimiento académico (Taras, 2005).

\section{Aplicaciones prácticas}

Con todas estas evidencias mostradas donde se analizó minuciosamente regiones, estructuras y funciones específicas del cerebro en niños, niñas y jóvenes de edad escolar por medio de técnicas de EEG y neuro-imagen (MRI). Así mismo, se describieron diferentes pruebas de laboratorio para evaluar la aptitud aeróbica. Todos estos trabajos de investigación fueron realizados en laboratorios sofisticados y costosos. Sin embargo, se puede lograr aplicaciones prácticas 
con test estandarizados de rendimiento cognitivo que evalúa la función ejecutiva considerando la edad, madures, grado escolar y otros factores de interés. También existen test indirectos que evalúan la capacidad aeróbica. De esta forma podríamos conocer los efectos, impactos, correlaciones o asociaciones que tiene la actividad física sobre el rendimiento académico sin necesidad de utilizar demasiados recursos económicos y así sugerir cambios o interacciones de actividad física vigorosa o moderada dependiendo de cada una de las características de los niños, niñas o jóvenes.

Por ello con este artículo sobre AF, cognición y rendimiento escolar esperamos incidir en las políticas públicas y en brindar una nueva visión a los profesores de educación física de clase directa, en la actitud de los padres de familia y en la participación de sociedad.

Se propone establecer estrategias de atención en horarios extraescolares para llevar a cabo la AF con niños niñas y jóvenes en áreas verdes, canchas de la escuela o institución a contra turno, inclusive escuelas abiertas los fines de semana, en instalaciones municipales, con ello se evitarán riesgos de salud física y mental de los niños, niñas y jóvenes con el propósito de que en el futuro existan adultos saludables.

\section{Futuras líneas de investigación}

Con la evidencia científica descrita nosotros consideramos que existe una línea de investigación definida sobre la intensidad en la clase de educación física, AF y rendimiento académico en niños, niñas y jóvenes. Considerando que altos niveles de aptitud aeróbica puede predecir rendimiento académico durante la niñez (Chaddock et al., 2012). Por ello seremos más específicos. Proponemos investigar sobre la aplicación y modulación de la AF, que permita analizar los efectos sobre el control cognitivo y ejecutivo. Creemos que se debe hacer un énfasis en investigar en un futuro a sujetos con niveles bajos de aptitud aeróbica y rendimiento académico. Para ello se propone aplicarles un método de incremento progresivo de AF y analizar sus efectos en el rendimiento académicos. Además, consideramos que se requiere mayor trabajo de investigación práctica y aplicada para conocer la asociación de la actividad física y la función ejecutiva en los niños, niñas o jóvenes. Por ello, hemos propuesto estas nuevas direcciones de investigación para el futuro.

\section{Agradecimientos}

Al Dr. Raúl Ortiz Pulido Profesor Investigador Titular C de tiempo completo de la Universidad Autónoma del Estado de Hidalgo por sus opiniones y observaciones realizadas a este trabajo.

\section{Referencias}

Ansari, D., \& Dhital, B. (2006). Age-related changes in the activation of the intrapariental sulcus during nonsymbolic magnitude processing: An event- related funtional magnetuc resonance imaging study. Journal of cognitive Neuroscience, 18(11), 1820-1828. doi:10.1162/ jocn.2006.18.11.1820
Arvidsson, D., Johannesson, E., Andersen, L. B., Karlsson, M., Wollmer, P., Thorsson, O., \& Dencker, M. (2018). A Longitudinal Analysis of the Relationships of Physical Activity and Body Fat With Nerve Growth Factor and Brain-Derived Neural Factor in Children. Journal of Physical Activity and Health, 20, 1-6. doi: 10.1123/ jpah.2017-0483

Bekhechi, A., \& K. Khiat, B. (2019) Impact of regular physical activity and sport on school performance among girls and boys aged between 6 and 10 years. Retos 36, 398402.

Best, J. (2010). Effects of physical activity on children's executive function: Contributions of experimental research on aerobic exercise. Developmental review, 30, 331-351. doi: 10.1016/j.dr.2010.08.001

Birnbaum, A. S., Lytle, L. A., Murray, D. M., Story, M., Perry, C. L., \& Boutelle, K. N. (2002). Survey development for assessing correlates of young adolescents' eating. American journal of health behavior, 26(4), 284295.

Blair, S. N., Kohl, H. W., Gordon, N. F., \& Paffenbarger Jr, R. S. (1992). How much physical activity is good for health? Annual review of public health, 13(1), 99-126.

Botvinick, M. M., Braver, T. S., Barch, D. M., Carter, C. S., \& Cohen, J. D. (2001). Conflict monitoring and cognitive control. Psychological review, 108(3), 624. doi: 10.1037/ 0033-295X.108.3.624

Brassen, S., Weber-Fahr, W., Sommer, T., Lehmbeck, J. T., \& Braus, D. F. (2006). Hippocampal-prefrontal encoding activation predicts whether words can be successfully recalled or only recognized. Behavioural brain research, 171(2), 271-278. doi: 10.1016/j.bbr.2006.04.002

Buck, S. M., Hillman, C. H., \& Castelli, D. M (2008). The relation of aerobic fitness to stroop task performance in preadolescent children. Med Sci Sport Exerc, 40(1), 16672. doi: 10.1249/mss.0b013e318159b035

Budde, H., Voelcker-Rehage, C., Pietraßyk-Kendziorra, S., Ribeiro, P., \& Tidow, G. (2008). Acute coordinative exercise improves attentional performance in adolecents. Neurosci Lett 441 (2) 219-23. doi: 10.1016/ j.neulet.2008.06.024

Cancela-Carral, J. M. C., Pérez, C.A., \& Espiño, M. J. S. (2016). The relationship between physical fitness and academic performance in Spanish secondary education students: A longitudinal study. Cultura Ciencia Deporte, 31(11), 7-16. doi: $10.12800 /$ ccd.v11i31.638

Casey, B. J., Jones, R. M., \& Hare, T. A. (2008). The adolescent brain. Annals of the New York Academy of Sciences, 1124(1), 111-126. doi: 10.1196/annals. 1440.010

Chaddock, L., Erickson, K. I., Prakash, R. S., Kim, J. S., Voss, M. W., VanPatter, M., ... \& Cohen, N. J. (2010a). A neuroimaging investigation of the association between aerobic fitness, hippocampal volume, and memory performance in preadolescent children. Brain research, 1358, 172-183. doi: 10.1016/j.brainres.2010.08.049

Chaddock, L., Erickson, K. I., Prakash, R. S., VanPatter, M., Voss, M. W., Pontifex, M. B., ... \& Kramer, A. F. (2010b). Basal ganglia volume is associated with aerobic fitness in preadolescent children. Developmental neuroscience, 32(3), 249-256. doi: 10.1159/000316648 
Chaddock, L., Pontifex, M. B., Hillman, C. H., \& Kramer,A. F. (2011a). A review of the relation of aerobic fitness and physical activity to brain structure and function in children. Journal of the International Neuropsychological Society, 17(6), 975-985. doi:10.1017/ S1355617711000567

Chaddock, L., Hillman, C. H., Buck, S. M., \& Cohen, N. J. (2011b). Aerobic fitness and executive control of relational memory in preadolescent children. Medicine \& Science in Sports \& Exercise, 43(2), 344-349. doi:10.1249/ MSS.0b013e3181e9af48

Chaddock, L., Erickson, K. I., Prakash, R. S., Voss, M. W., VanPatter, M., Pontifex, M. B., ... \& Kramer,A. F. (2012). A functional MRI investigation of the association between childhood aerobic fitness and neurocognitive control. Biological psychology, 89(1), 260-268. doi: 10.1016/ j.biopsycho.2011.10.017

Chaddock-Heyman, L., Erickson, K. I., Kienzler, C., King, M., Pontifex, M. B., Raine, L. B., ... \& Kramer, A. F. (2015). The role of aerobic fitness in cortical thickness and mathematics achievement in preadolescent children. PLOS One, 8, 1-11. doi: 10.1371/journal.pone.0134115

Chomitz, V. R., Slining, M. M., McGowan, R. J., Mitchell, S. E., Dawson, G. F., \& Hacker, K.A. (2009). Is there a relationship between physical fitness and academic achievement? Positive results from public school children in the northeastern United States. Journal of School Health, 79(1),30-37. doi:10.1111/j.1746-1561.2008.00371.x

Coe, D. P., Pivarnik, J. M., Womack, C. J., Reeves, M. J., \& Malina, R. M. (2006). Effect of physical education and activity levels on academic achievement in children. Medicine \& Science in Sports \& Exercise, 38(8), doi: 1515-1519.10.1249/01.mss.0000227537.13175.1b

Cohen, N. J., Ryan, J., Hunt, C., Romine, L., Wszalek, T., \& Nash, C (1999). Hippocampal system and declarative (relational) memory: summarizing the data from functional neuroimaging studies. Hippocampus, 9(1),83-98. doi: 10.1002/(SICI) 1098-1063(1999)9: 1<83::AIDHIPO9>3.0.CO;2-7

Cotman, C.W., \& Berchtold, N. C. (2002). Exercise: a behavioral intervention to enhance brain health and plasticity. Trends in neurosciences, 25(6), 295-301. doi: 10.1016/ S0166-2236(02)02143-4

Cotman, C. W., Berchtold, N. C., \& Christie, L. A. (2007). Exercise builds brain health: key roles of growth factor cascades and inflammation. Trends in neurosciences, 30(9), 464-472. doi: 10.1016/j.tins.2007.06.011

Culham, J. C., \& Kanwisher, N. G. (2001). Neuroimaging of cognitive functions in human parietal cortex. Current opinion in neurobiology, 11(2),157-163. doi:10.1016/ S0959-4388(00)00191-4

Daniels, S. R., \& Greer, F. R. (2008). Lipid screening and cardiovascular health in childhood. Pediatrics, 122(1), 198-208.

Davis, C. L., Tomporowski, P. D., McDowell, J. E., Austin, B. P., Miller, P. H., Yanasak, N. E., ... \& Naglieri, J. A. (2011). Exercise improves executive function and achievement and alters brain activation in overweight children: a randomized, controlled trial. Health Psychology, 30(1), 91-98. doi:91. 10.1037/a0021766
Diamond, A., \& Lee, K. (2011). Interventions shown to aid executive function development in children 4 to 12 years old. Science, 333(6045), 959-954. doi: 10.1126/ science. 1204529

Dietz, W. H. (1996). The role of lifestyle in health: the epidemiology and consequences of inactivity. Proceedings of the Nutrition Society, 55(03), 829-840.

DuPaul, G. J., Power, T. J., Anastopoulos, A. D., \& Reid, R. (1998). ADHD Rating Scale-IV: Checklists, norms, and clinical interpretation. Guilford Press.

Ekblom-Bak, E., Ekblom, O., Andersson, G., Wallin, P., \& Ekblom, B. (2018). Physical Education and Leisure-Time Physical Activity in Youth Are Both Important for Adulthood Activity, Physical Performance, and Health. Journal of Physical Activity and Health, 15(9), 661-670 doi: 10.1123/jpah.2017-0083

Esteban-Cornejo, I., Hallal, P. C., Mielke, G. I., Menezes, A. M., Gonçalves, H., Wehrmeister, F., ... \& Rombaldi, A. J. (2015). Physical activity throughout adolescence and cognitive performance at 18 years of age. Medicine and science in sports and exercise, 47(12), 2552-2557. doi: 10.1249/MSS.0000000000000706

Ferris, L. T., Williams, J. S., \& Shen, C. L. (2007). The effect of acute exercise on serum brain-derived neurotrophic factor levels and cognitive function. Medicine \& Science in Sports \& Exercise, 728-734. doi: 10.1249/ mss.0b013e31802f04c7

Flores, E., Maureira, F., Diaz, H., Navarro, B., Gavotto, O., \& MatheuA. (2019) Effects of a session of physical exercise on the neurophysiological activity during the resolution of a test of selective attention. Retos 36, 391-397.

Fraile-García, J., Terejo-Gonzalez, C.M., Esteban-Cornejo, I., \& Veiga, O. (2019). Association between enjoyment, motor selfefficacy, physycal activity and performance. Retos 3658-63

Gelabert, J., Muntaner-Mas, A., \& Palou, P. (2019)Association between active commuting to school and body composition and academic achievement in schoolchildren aged 10-12 years. Retos 36, 376-383.

Hallal, P. C., Azevedo, M. R., Reichert, F. F., Siqueira, F. V., Araújo, C. L., \& Victora, C. G. (2005). Who, when, and how much?: Epidemiology of walking in a middle-income country. American journal of preventive medicine, 28(2), 156-161.

Hillman, C. H., Erickson, K. I., \& Kramer, A. F. (2008). Be smart, exercise your heart: exercise effects on brain and cognition. Nature reviews neuroscience, 9(1), 58-65. doi: 10.1038/nrn2298

Hillman, C. H., Buck, S. M., Themanson, J. R., Pontifex, M. B., \& Castelli, D. M.(2009). Aerobic fitness and cognitive development: Event-related brain potential and task performance indices of executive control in preadolescent children. Developmental psychology, 45(1), 114. doi: 10.1037/a0014437

Hillman, C. H., Pontifex, M. B., Castelli, D. M., Khan, N. A., Raine, L. B., Scudder, M. R., ... \& Kamijo, K. (2014).Effects of the FITKids randomized controlled trial on executive control and brain function. Pediatrics, 134(4), doi: 10631071. 10.1542/peds.2013-3219

Hollis, J. L., Williams, A. J., Sutherland, R., Campbell, E., 
Nathan, N., Wolfenden, L., ... \& Wiggers, J (2016). A systematic review and meta-analysis of moderate-tovigorous physical activity levels in elementary school physical education lessons. Preventive medicine, 86, 34-54.

Hollis, J. L. Sutherland, R., Williams, A. J., Campbell, E., Nathan, N., Wolfenden, L., ... \& Wiggers, J. (2017). A systematic review and meta-analysis of moderate-to-vigorous physical activity levels in secondary school physical education lessons. International Journal of Behavioral Nutrition and Physical Activity, 14(1), 52.

Janssen, I., \& LeBlanc, A. G. (2010). Systematic review of the health benefits of physical activity and fitness in schoolaged children and youth. International Journal of Behavioral nutrition and physical activity, 7(40), 1-16.

Kaufman, A. S. (1990). Kaufman briefintelligence test: KBIT. Circle Pines, MN: AGS, American Guidance Service.

Keeley, T. J., \& Fox, K. R. (2009). The impact of physical activity and fitness on academic achievement and cognitive performance in children. International Review of Sport and Exercise Psychology, 2(2), 198-214. doi: 10.1080/17509840903233822

Khan, N. A., \& Hillman, C.H. (2014). The relation of childhood physical activity and aerobic fitness to brain function and cognition: a review. Pediatric exercise science, 26(2), 138-146. doi:10.1123/pes.2013-0125

Krabbe, K. S., Nielsen, A. R., Krogh-Madsen, R., Plomgaard, P., Rasmussen, P., Erikstrup, C., ... \& Secher, N. H. (2007). Brain-derived neurotrophic factor (BDNF) and type 2 diabetes. Diabetologia, 50(2), 431-438. doi: 10.1007/ s00125-006-0537-4

LeBlanc, M. M.., Martin, C. K., Han, H., Newton Jr, R., Sothern, M., Webber, L. S., ... \& Williamson, D.A. (2012).Adiposity and physical activity are not related to academic achievement in school-aged children. Journal of developmental and behavioral pediatrics, 33(6), 486494. doi: 10.1097/DBP.0b013e31825b849e

Lee, I. M., Shiroma, E. J., Lobelo, F., Puska, P., Blair, S. N., Katzmarzyk, P. T., \& Lancet Physical Activity Series Working Group. (2012). Effect of physical inactivity on major non-communicable diseases worldwide: an analysis of burden of disease and life expectancy. The lancet, 380(9838), 219-229.

Leger, L.A., Mercier, D., Gadoury, C., \& Lambert, J. (1988). The multistage 20 metre shuttle run test for aerobic fitness. Journal of sports sciences, 6(2), 93-101.

Lu, B., Pang, P. T., \& Woo, N. H. (2005). The yin and yang of neurotrophin action. Nature Reviews Neuroscience, 6(8), 603-613. doi: 10.1038/nrn1726

Maguire, E. A., Frith, C. D., \& Morris, R. G. M. (1999). The functional neuroanatomy of comprehension and memory: the importance of prior knowledge. Brain, 122(10), 18391850. doi: $10.1093 /$ brain/122.10.1839

Marshall, S. J., Biddle, S. J., Gorely, T., Cameron, N., \& Murdey, I. (2004). Relationships between media use, body fatness and physical activity in children and youth: a metaanalysis. International journal of obesity, 28(10), 12381246.

McDonald, D. M., \& Choyke, P. L. (2003). Imaging of angiogenesis: from microscope to clinic. Nature medici- ne, 9(6), 713. doi: 10.1038/nm0603-713

Meyer, D. E., \& Kieras, D. E. (1997). A computational theory of executive cognitive processes and multiple-task performance: Part I. Basic mechanisms. Psychological review, 104(1), 3-65. doi: 10.1037/0033-295X.104.1.3

Mura, G., Vellante, M., Egidio Nardi, A., Machado, S., \& Giovanni Carta, M.(2015). Effects of school-based physical activity interventions on cognition and academic achievement: a systematic review. CNS \& Neurological Disorders-Drug Targets (Formerly Current Drug Targets-CNS \& Neurological Disorders), 14(9), 11941208.

Murer, M. G., Yan, Q., \& Raisman-Vozari, R. (2001). Brainderived neurotrophic factor in the control human brain, and in Alzheimer's disease and Parkinson's disease. Progress in neurobiology, 63(1), 71-124. doi: 10.1016/ S0301-0082(00)00014-9

Neeper, S. A., Góauctemez-Pinilla, F., Choi, J., \& Cotman, C. (1995). Exercise and brain neurotrophins. Nature, 373(6510), 109. doi: 10.1038/373109a0

Ng, S. W., \& Popkin, B. M. (2012). Time use and physical activity: a shift away from movement across the globe. Obesity Reviews, 13(8), 659-680

Oliver, M., Schofield, G. M., \& Kolt, G. S. (2007). Physical activity in preschoolers. Sports medicine, 37(12), 10451070.

Palmer, T. D., Willhoite, A. R., \& Gage, F. H. (2000). Vascular niche for adult hippocampal neurogenesis. Journal of Comparative Neurology, 425(4), 479-494. doi: 10.1002/ 1096-9861(20001002)425:4<479::AID-CNE2>3.0.CO;2-3

Pan, W., Banks, W.A., Fasold, M. B., Bluth, J., \& Kastin, A. J. (1998). Transport of brain-derived neurotrophic factor across the blood-brain barrier. Neuropharmacology, 37(12), 1553-1561. doi: 10.1016/S0028-3908(98)00141-5

Paffenbarger Jr, R. S. Hyde, R.T., Wing, A.L., \& Hsienh, C.C. (1986). Physical activity, all-cause mortality, and longevity of college alumni. New England journal of medicine, 314(10), 605-613. Doi 10.1056/NEJM198603063141003

Park, H., \& Poo, M. M. (2013). Neurotrophin regulation of neural circuit development and function. Nature Reviews Neuroscience, 14(1), 7-23. doi: 10.1038/nrn3379

Pereira, A. C., Huddleston, D. E., Brickman, A. M., Sosunov, A. A., Hen, R., McKhann, G. M., ... \& Small, S.A. (2007). An in vivo correlate of exercise-induced neurogenesis in the adult dentate gyrus. Proceedings of the National Academy of Sciences, 104(13), 5638-5643. doi: 10.1073/ pnas.0611721104

Pesce, C., Crova, C., Cereatti, L., Casella, R., \& Bellucci, M., (2009) Physycal activity and mental performance in preadolecents: Effects of acute exercise on freerecall memory. Mental Health and Physycal Activity. 2(1) 1622 doi.org/10.1016/j.mhpa2009.02001

Plowman, S.A. \& Meredith, M.D.(Eds.). (2013). Fitnessgram/ Activitygram Reference Guide (4th Edition). Dallas, TX: The Cooper Institute.

Polich, J., Ladish, C., \& Burns, T. (1990). Normal variation of P300 in children: age, memory span, and head size. International Journal of Psychophysiology, 9(3), 237-248.

Rasberry, C. N., Lee, S. M., Robin, L., Laris, B. A., Russell, L. 
A., Coyle, K. K., \& Nihiser, A. J. (2011). The association between school-based physical activity, including physical education, and academic performance: a systematic review of the literature. Preventive medicine, 52, S10-S20. doi: 10.1016/j.ypmed.2011.01.027

Rivera, S. M., Reiss, A. L., Eckert, M. A., \& Menon, V (2005). Developmental changes in mental arithmetic: evidence for increased functional specialization in the left inferior parietal cortex. Cerebral cortex, 15(11), 1779-1790. doi: 10.1093/cercor/bhi055

Roberts, C. K., Freed, B., \& McCarthy, W. J. (2010). Low Aerobic Fitness and Obesity Are Associated with Lower Standardized test scores in children. The Journal of pediatrics, 156(5), 711-718. doi: 10.1016/ j.jpeds.2009.11.039

Ruiz-ArizaA., De la Torre-Cruz, M.J., Suaréz-Manzano, S., \& Martínez-López E.J. (2019). Support Towards physical activity and academic performance regardless of parental socio-educational. Retos, 35, 208-212.

Rohde, T. E., \& Thompson, L.A. (2007). Predicting academic achievement with cognitive ability. Intelligence, 35(1), 83-92. doi: 10.1016/j.intell.2006.05.004

Rosa, A., Garcia. E., \& Carrillo, P.J. (2019a). Relationship between aerobic capacity and level of attention in primary school children. Retos, 35, 36-41

Rosa, A., Garcia. E., \& Carrillo, P.J. (2019b). Aerobic capacity and academic performance in primary schoolchildren. Retos, 35, 351-354

Schinder, A. F., \& Poo, M. M. (2000). The neurotrophin hypothesis for synaptic plasticity. Trends in neurosciences, 23(12), 639-645. doi: 10.1016/S01662236(00)01672-6

Schmidt, H. D., \& Duman, R. S. (2010). Peripheral BDNF produces antidepressant-like effects in cellular and behavioral models. Neuropsychopharmacology, 35(12), 2378.

Shephard, R. (1997). Curricular physical activity and academic performance. Pediatric exercise science, 2(9), 113-126. doi: 10.1123/pes.9.2.113

Shvartz, E., \& Reibold, R. C. (1990). Aerobic fitness norms for males and females aged 6 to 75 years: a review. Aviation, space, and environmental medicine, 61(1), 3-11.

Sibley, B. A., \& Etnier, J. L. (2003). The relationship between physical activity and cognition in children: a metaanalysis. Pediatric exercise science, 15(3), 243-256. doi: 10.1123/pes.15.3.243

Singh, A., Uijtdewilligen, L., Twisk, J. W., Van Mechelen, W., \& Chinapaw, M. J. (2012). Physical Activity and performace at school: A systematic review of the literatura including a methodological quality assessment. Arch Pediatr Adolec Med, 166(1), 49-55. doi: 10.1001/ archpediatrics.2011.716

Sirard, J. R. (2005). Calibration and evaluation of an objective measure of physical activity in preschool children. Journal of physical activity and health, 2(3), 345-357.

Stathopoulou, G., Powers, M. B., Berry, A. C., Smits, J. A., \& Otto, M. W. (2006). Exercise interventions for mental health: a quantitative and qualitative review. Clinical Psychology: Science and Practice, 13(2), 179-193.
Strong, W. B., Malina, R. M., Blimkie, C. J., Daniels, S. R., Dishman, R. K., Gutin, B., ... \& Rowland, T. (2005). Evidence based physical activity for school-age youth. The Journal of pediatrics, 146(6), 732-737. doi: 10.1111/j.14682850.2006.00021.x

Syväoja, H., Kantomaa, M. T., Ahonen, T., Hakonen, H., Kankaanpää,A., \& Tammelin, T.H.(2013). Physical activity, sedentary behavior, and academic performance in Finnish children. Medicine and science in sports and exercise, 45(11). doi: 10.1249/MSS.0000000000000335

Szuhany, K. L., Bugatti, M., \& Otto, M. W. (2015). A metaanalytic review of the effects of exercise on brain-derived neurotrophic factor. Journal of psychiatric research, 60, 56-64. doi: 10.1016/j.jpsychires.2014.10.003

Taras, H. (2005). Physical activity and student performance at school. Journal of school health, 75(6), 214-218. doi: 10.1111/j.1746-1561.2005.tb06675.x

Taylor, S. J., Whincup, P. H., Hindmarsh, P. C., Lampe, F., Odoki, K., \& Cook, D. G. (2001). Performance of a new pubertal self-assessment questionnaire: a preliminary study. Paediatric and perinatal epidemiology, 15(1), 8894.

Tremblay, M. S., Inman, J. W., \& Willms, J. D. (2000). The relationship between physical activity, self-esteem, and academic achievement in 12-year-old children. Pediatric exercise science, 12(3), 312-323. doi: 10.1123/pes.12.3.312

Tammelin, T., Ekelund, U., Remes, J., \& Näyhä, S. (2007). Physical activity and sedentary behaviors among Finnish younth. Medicine and science in sports and exercise, 39(7), 1067-1074.

Trudeau, F., \& Shephard, R. J.(2008). Physical education, school physical activity, school sports and academic performance. International Journal of Behavioral Nutrition and Physical Activity, 5(1), 10. doi: 10.1186/1479-5868-510

Utter, A. C., Robertson, R. J., Nieman, D. C., \& Kang, J. I. E. (2002). Children's OMNI Scale of Perceived Exertion: walking/running evaluation. Medicine \& Science in Sports \& Exercise.

Van Praag, H. (2008). Neurogenesis and exercise: past and future directions. Neuromolecular medicine, 10(2), 128140. doi: 10.1007/s12017-008-8028-z

Winter, B., Breitenstein, C., Mooren, F. C., Voelker, K., Fobker, M., Lechtermann, A., ... \& Knecht, S. (2007). High impact running improves learning. Neurobiol Learn Mem, 87(4), 597-609.

Wu, C. T., Pontifex, M. B., Raine, L. B., Chaddock, L., Voss, M. W., Kramer, A. F., \& Hillman, C. H. (2011). Aerobic fitness and response variability in preadolescent children performing a cognitive control task. Neuropsychology, 25(3), 333.

Zoladz, J. P \& Pilc, J. (2010). The effect of physical activity on the brain derived neurotrophic factor: from animal to human studies. Journal of Physiology and Pharmacology, 61(5), 533-541. doi: 10.1016/ j.nlm.2006.11.003 